\title{
Thermal Probe Measurements of Energy Flux onto a Substrate during ICP Assisted Sputter-Deposition
}

\author{
Hiroaki Kitagawa, Ryoji Kan, \\ Kenji Mine, Masanori Shinohara \\ Graduate School of Science and Technology \\ Nagasaki University \\ Nagasaki, Japan
}

\author{
Yoshinobu Matsuda \\ Department of Electrical and Electronic Engineering \\ Nagasaki University \\ Nagasaki, Japan \\ ymat@nagasaki-u.ac.jp
}

\begin{abstract}
Substrate energy flux in the inductively coupled plasma (ICP) assisted magnetron discharge has been measured by using a home-made thermal probe. The energy flux depends predominantly on the ICP power. In addition, substrate bias dependence of the substrate energy flux has been measured. As a result, it has been confirmed that the measured heat influx in an ICP is in good agreement with the theoretically calculated value within $30 \%$ error. Thus, substrate energy flux during the ICP assisted sputtering is quantitatively clarified.
\end{abstract}

Keywords-Thermal probe; energy flux; inductively coupled plasma; transparent conductive film

\section{INTRODUCTION}

Tin-doped indium oxide (ITO) has been widely used as a transparent conductive film. The reason why the ITO has been widely used is that a large area sputter-deposition of transparent conducting films of low-resistivity and high optical transmittance can be made with low-cost and good reproducibility. However, alternative materials to ITO have been anticipated, because the indium is a rare metal and ITO powder has toxicity. Recently, aluminum doped zinc oxide (AZO) received attention as an alternative material. The AZO is stable in the reduction atmosphere, low-cost, and harmless in the human body. However, fabrication process is unstable and its reproducibility is scarce. Therefore, it is necessary to establish the deposition process with high reliability. In addition, a further improvement of film quality is necessary. Therefore we have been studying deposition process of AZO films by using inductively coupled plasma (ICP) assisted magnetron sputtering. As a result, we can obtain good quality (high crystallinity, high conductivity and high optical transmittance) AZO thin films, in particular when the sputtering is assisted with increased ICP power [1]. However, not only the relation between the plasma parameters and thin film properties but also the role of ICP assist power on them are not clarified yet. Therefore, the grasp of the energy flux onto a substrate during the ICP assisted spattering is very important.

The energy flux to the substrate can be directly measured by using a thermal probe (TP) [2-10] in different ways. One is the method that uses the difference in the rising and falling rate of TP temperature during the plasma on and off periods, in which the heat capacity of the TP has to be calibrated. Another one is the method that uses the temperature difference in the TP in the thermal equilibrium condition, in which two thermocouples have to be set apart at two different positions of the probe.

Energy flux onto a substrate has been measured with different TPs by many researchers in the RF plasma [2-7], $\mathrm{RF}$ magnetron plasma [8,9], and DC grow discharge plasma [10] in the past. However, no measurement of the energy flux onto the substrate during the ICP assisted sputtering has been done as far as we know so far. Therefore, we have measured the energy flux onto the substrate during the ICP assisted sputtering. For the first trial of measuring the energy flux, we adopted here the temperature equilibrium type TP. This paper reports the first experimental results of the measurement of energy flux onto the substrate during the ICP assisted sputtering.

\section{EXPERIMENTAL}

The principle of measuring the energy flux that uses a temperature equilibrium type TP is as follows. Let the side of TP be completely insulated from heat and one end of TP be connected to the heat sink. Furthermore, we assume the energy flux flows in from another end of TP with a constant cross section, and the thermal conduction occurs only in the longer direction. When two small thermocouples are installed separately in the different axial locations with a gap distance of $\Delta \mathrm{x}$ on the TP, the inflow energy flux (energy per unit area per second) $Q_{\text {in }}$ is given by the equation

$$
Q_{i n}=-k \frac{\Delta T}{\Delta x}
$$

where $\Delta \mathrm{x}$ is the axial distance between the two thermocouples, $\Delta \mathrm{T}$ is the temperature difference between the two thermocouples in the temperature equilibrium condition, and $\mathrm{k}$ is the heat conductivity of the TP head.

Figure 1 shows the schematic of the TP made in this work. The TP is composed of a copper plate $(0.1 \mathrm{~mm}$ in thickness) facing to the plasma, a ceramics rod $(6 \mathrm{~mm}$ in diameter, $10 \mathrm{~mm}$ in length) producing temperature gradient, an aluminum rod (6mm in diameter, $350 \mathrm{~mm}$ in length) connected to the outer heat sink, and outer tubes (Teflon, alumina, and stainless steel tubes) for thermal and electric insulation, which prevents the energy flux from coming in from the side of the TP. The copper plate, ceramic rod and aluminum rod were tightly bonded. A tip of first thermocouple was attached on the ceramics rod at the distance of $6 \mathrm{~mm}$ from the joint plane between the ceramics 
and the aluminum rods. The tip of the second thermocouple was attached on the aluminum rod with a distance of $10 \mathrm{~mm}$ from the joint plane. The other end of the aluminum rod was water-cooled outside the vacuum vessel. The outer stainless steel tube for heat insulation was grounded to cut the electrical coupling between the TP and plasmas.

Two different shaped copper plates were tested in the experiment: a square copper plate $(10 \mathrm{~mm} \times 10 \mathrm{~mm}, 0.1 \mathrm{~mm}$ in thickness) and a circle copper plate $(3.5 \mathrm{~mm}$ in radius, $0.1 \mathrm{~mm}$ in thickness). In the experiment, the square copper plate having an area larger than the cross section of the ceramic rod was first used to make the greatest difference in the equilibrium temperature between the two ends of ceramics rod, and to improve the signal to noise ratio in the temperature measurement. The square copper plate worked well when it was used at a floating potential, but did not work correctly when it was biased due to the occurrence of abnormal micro-arcing. Thus, the square copper plate was replaced with the circle copper plate, which was connected with a lead wire and used as a biased electrode.

The most thermal gradient appears on the ceramic rod that has a thermal conductivity of $1.6 \mathrm{~W} /(\mathrm{m} \cdot \mathrm{K})$. The length of the ceramics and the spacing of the two TP tips were determined by the tradeoff between the requirements of smaller thermal time constant of the ceramic rod and that of larger difference in temperature at both the ends of the ceramic rod. The thermal time constant is expressed as follows:

$$
\tau=C R=\rho c V \frac{L}{k S}=\frac{\rho c}{k} L^{2},
$$

where $C$ is thermal capacity, $R$ is thermal resistance, $\rho$ $\left[\mathrm{kg} / \mathrm{m}^{3}\right]$ is mass density, $c[\mathrm{~J} /(\mathrm{kg} \cdot \mathrm{K})]$ is specific heat, $k$ $[\mathrm{W} /(\mathrm{m} \cdot \mathrm{K})]$ is thermal conductivity, $V\left[\mathrm{~m}^{3}\right]$ is volume, $S$ $\left[\mathrm{m}^{2}\right]$ is cross section area, $L[\mathrm{~m}]$ is length of the TP body.

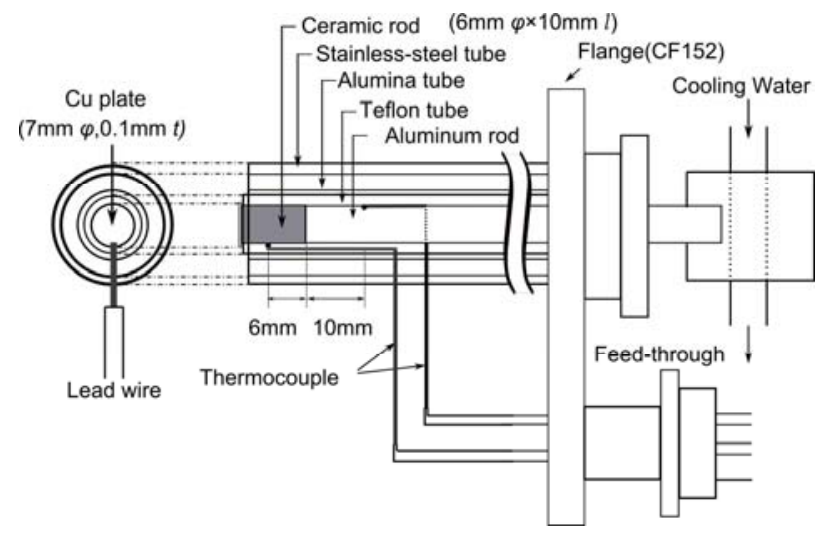

Figure 1. Schematic of thermal probe.

The shorter the length of ceramic rod is, the smaller the thermal time constant is. The quick response of the probe is favorable to improve the measurement efficiency. On the other hand, the longer the length of the ceramic rod is, the larger the temperature difference along the ceramic rod is as shown in eq.(1). The aluminum rod has large thermal conductivity and the temperature gradient in the aluminum rod is very small. It mostly works as a heat sink because the end of the aluminum rod was water-cooled.

Figure 2 shows the experimental setup for the energy flux measurement in ICP assisted sputter-deposition. A vacuum chamber $(300 \mathrm{~mm}$ in diameter and $300 \mathrm{~mm}$ in height) was attached with a 3inch DC planar magnetron, an argon gas supply system, and a pumping system (turbo molecular pump and rotary pump combination).A disk target of $\mathrm{ZnO}: \mathrm{Al}_{2} \mathrm{O}_{3}(2 \mathrm{wt} \%)$ of $60 \mathrm{~mm}$ diameter and $6 \mathrm{~mm}$ thick was used as target. Between these diode electrodes, a single turn coil antenna of $100 \mathrm{~mm}$ diameter was installed and used for the production of $13.56 \mathrm{MHz}$ inductively coupled plasma. The antenna was covered with insulator and water-cooled. The distance from the target to the RF coil and the distance from the RF coil to the substrate were both $40 \mathrm{~mm}$. The TP was set $8 \mathrm{~cm}$ from the target surface in the axial direction, and $3 \mathrm{~cm}$ from the center axis of magnetron. An argon gas was introduced at a flow rate of $50 \mathrm{sccm}$ by a mass flow controller. The operation gas pressure was set to $30 \mathrm{mT}$ Torr by adjusting the main valve. After that, cooling water was flowed through the target, ICP antenna and the TP. The ICP electric power was turned on for some period until the thermal equilibrium was attained. Then, ICP power was turned off. Temperatures at two different positions were measured by two thermocouples installed in a TP, and were displayed on digital multi meters, then temperatures were recorded on a personal computer. To clean the copper plate surface, the bias voltage of $-40 \mathrm{~V}$ was applied for ten minutes every time in the measurement of bias dependence.

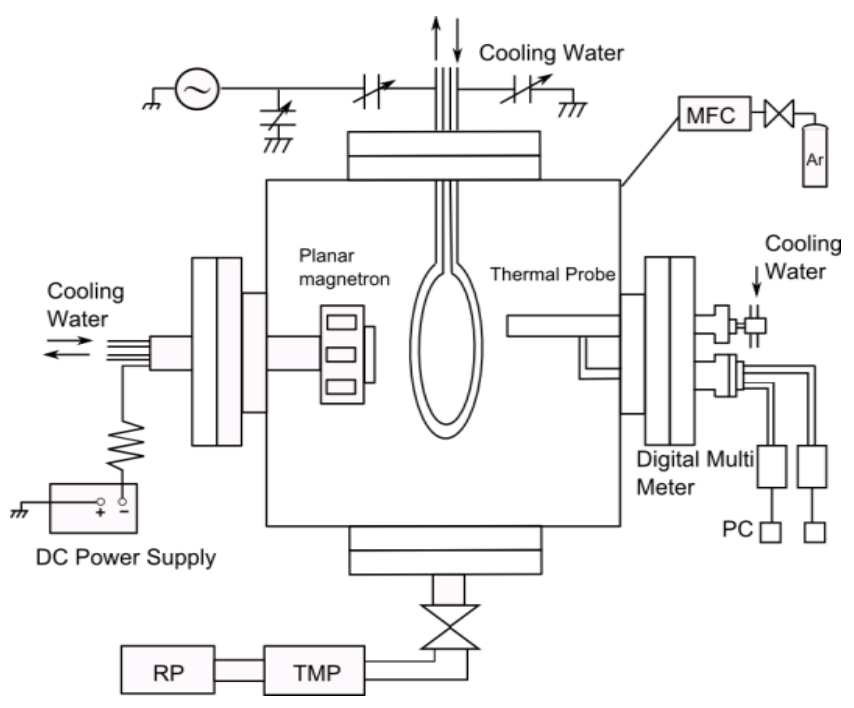

Figure 2. Experimental setup for the energy flux measurement in ICP assisted sputter-deposition. 


\section{RESULTS AND DISCUSSION}

\section{A. Basic operation characteristic of thermal probe}

Figure 3 shows the temporal change of difference in temperature measured by the two thermocouples on the TP for the ICP RF power $200 \mathrm{~W}$ and the target power $0 \mathrm{~W}$. The ICP was turned off in 500s. The data shown in Fig. 3 indicates that the difference in temperature is mostly saturated with a time constant $\tau$ of 90 seconds and the thermal equilibrium condition is reached at the time after four or five $\tau$ seconds. It is noted that the time constant $\tau$ calculated by using the expression (2) is 130s. Thus, the experimental thermal time constant is close to the calculated one. The energy flux onto the TP is evaluated to be $1600 \mathrm{~W} / \mathrm{m}^{2}$ by substituting the saturated temperature difference (21degree celcius) obtained at the time after $5 \tau$ for the equation (1).

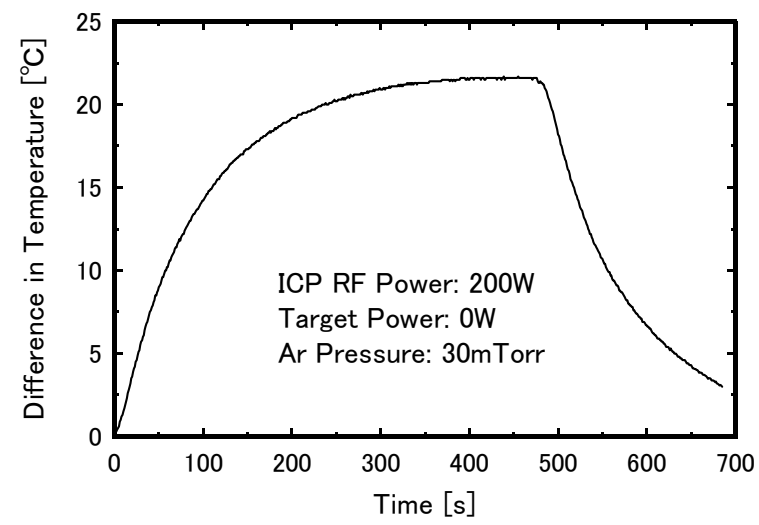

Figure 3. Temporal change in temperature difference obtained by a thermal probe during on and off period of $200 \mathrm{~W}$ ICP.

\section{B. Target power and ICP RF power dependence of energy} flux.

Figure 4 shows the target power dependence of energy flux when only the DC planar magnetron (PM) operates. We found that energy flux linearly increases with increasing target power.

Figure 5 shows ICP RF power dependence of energy flux when only the ICP operates. We found that energy flux also linearly increases with increasing ICP RF power. By comparing the energy fluxes of Figure 4 and Figure 5 at the same input power, it is noted that the value of the energy flux when only the ICP operates is about six times larger than that of the energy flux when only the DC PM operates, This is explained by the difference in their spatial distribution. The spatial distribution of ICP is so large that the TP is exposed to the high density ICP; on the other hand, the spatial distribution of PM is localized so close to the target that the TP is exposed to the low density edge of PM plasma.

\section{Energy flux during ICP assisted sputter-deposition}

Figure 6 shows ICP power dependence of energy flux when DC PM power is $0 \mathrm{~W}, 40 \mathrm{~W}$, and $50 \mathrm{~W}$, i.e. in the case of ICP assisted sputtering. Figure 6 indicates that the energy flux depends more on the ICP RF power and less on the PM target power. The energy flux increases in proportion to the increase in PM target power at the ICP RF power of $0 \mathrm{~W}$. On the other hand, at the ICP RF power of $200 \mathrm{~W}$, the energy flux with PM target power of 40 and $50 \mathrm{~W}$ are less than that with PM target power of $0 \mathrm{~W}$. Thus, the energy flux in the ICP assisted sputtering is not a simple superposition of the energy flux for the pure PM discharge and the one for the pure ICP. This can be explained as follows. Increasing target power leads to an accumulation of many sputtered particles. These particles would be easily ionized in the ICP, because the ionizing potential ( $\mathrm{Al}: 5.986 \mathrm{eV}, \mathrm{Zn}: 9.394 \mathrm{eV}$ ) of the metallic atom is lower than that of $\mathrm{Ar}(15.8 \mathrm{eV})$. As a result, the electron density in ICP would increase and its electron temperature would decrease. The decrease in the electron temperature would decrease the potential difference between the space potential of ICP and the substrate potential. Thus, an increase in PM target power would decrease the positive ion flux onto the substrate. On the other hand, the energy of high-energy particles onto the substrate would decrease with increasing the ICP RF power as far as a constant PM target power is injected, since the target current increases and the target voltage decreases with increasing ICP RF power at the constant PM target power.

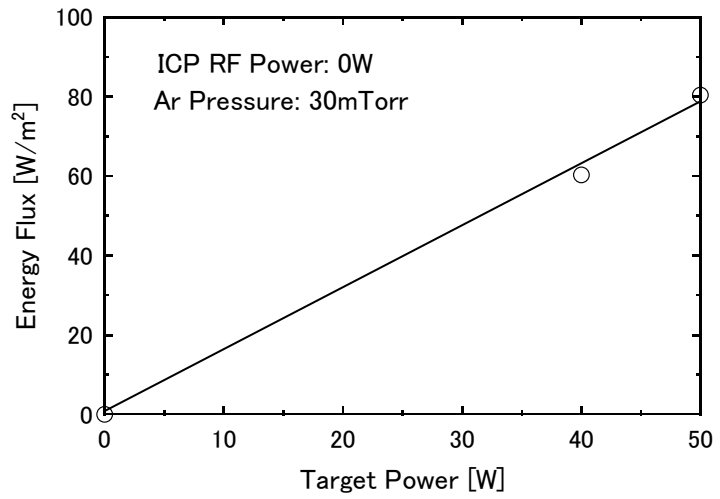

Figure 4. Target power dependence of energy flux (operation with PM alone).

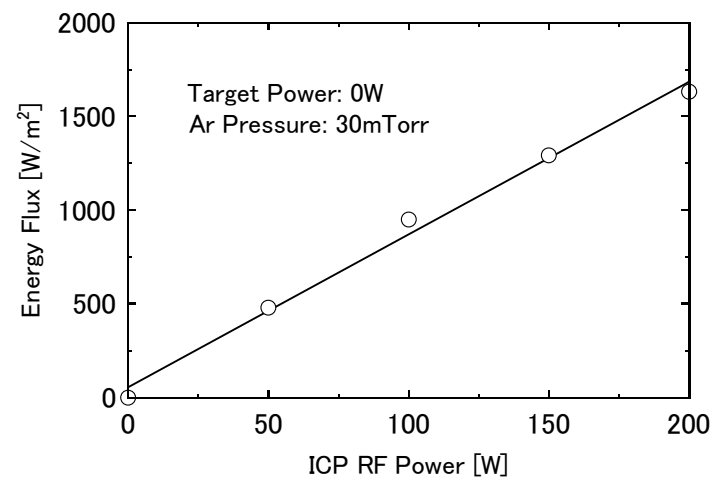

Figure 5. ICP RF power dependence of energy flux (operation with ICP alone ). 


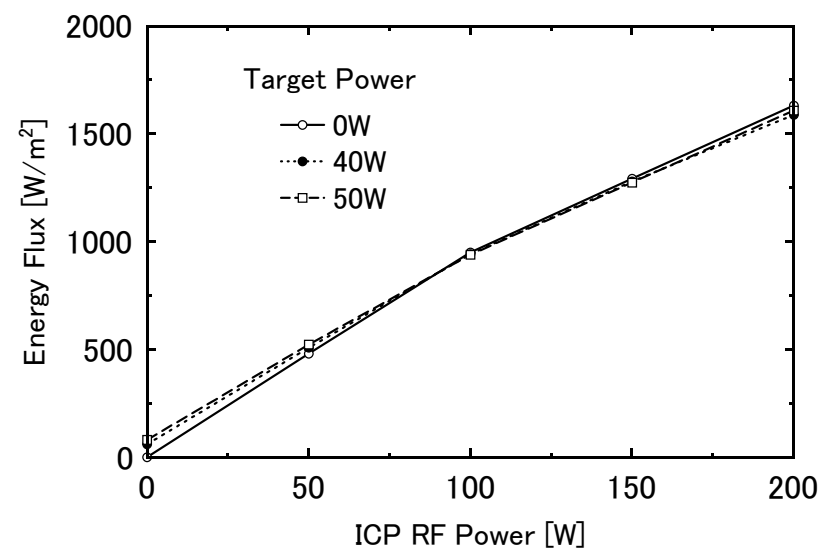

Figure 6. ICP RF power dependence of energy flux (operation with ICP assisted PM sputtering).

\section{The substrate bias dependency of the energy flux}

The change in energy flux against substrate bias was investigated experimentally and theoretically. Experimental values measured at various substrate bias and at various combinations of ICP RF power and PM target power: $(100,0),(100,40),(200,0)$, and $(200,40)$ are plotted with symbols of white triangle, black triangle, white circle, and black circle, respectively.

According to Kersten et al., the total energy flux onto a substrate $J$ is expressed as the addition of the contribution $J_{\mathrm{i}}$ by the positive ions, the contribution $J_{\mathrm{e}}$ by the negative electrons, the contribution $J_{\text {rec }}$ by the recombination of positive ions and electrons at the substrate surface, the contribution $J_{\mathrm{n}}$ by neutral species (heat of adsorption or condensation, excitation energy, kinetic energy), and the contribution of heat radiation $J_{\text {rad }}$ as shown in equation (3). In this work, $J_{\text {rad }}$ was negligible because of low substrate temperature and $J_{\mathrm{n}}$ was ignored because of complexity of treatment. Each contribution of $J_{\mathrm{i}}, J_{\mathrm{e}}$ and $J_{\text {rec }}$ is given by expressions (4), (5) and (6).

$$
\begin{aligned}
& J=J_{i}+J_{e}+J_{r e c}+J_{n}+J_{r a d}, \\
& J_{i}=n_{e} \sqrt{\frac{k T_{e}}{m_{i}}} \exp (-0.5) e_{0}\left(V_{p l}-V_{s}\right), \\
& J_{e}=n_{e} \sqrt{\frac{k T_{e}}{2 \pi m_{e}}} \exp \left(-\frac{e_{0} V_{\text {bias }}}{k T_{e}}\right) 2 k T_{e}, \\
& J_{r e c}=j_{i} E_{r e c} .
\end{aligned}
$$

Here, $n_{\mathrm{e}}$ is electron density, $k T_{\mathrm{e}}$ the electron temperature, $m_{\mathrm{i}}$ the mass of ion, $m_{\mathrm{e}}$ the mass of electron, $e_{0}$ the base of natural logarithm, $V_{\mathrm{pl}}$ the plasma space potential, $V_{\mathrm{s}}$ the substrate potential, $V_{\text {bias }}\left(=V_{\mathrm{pl}}-V_{\mathrm{s}}\right)$ the potential fall from plasma to the substrate, and $E_{\text {rec }}$ the recombination energy of a positive ion.

Theoretical energy flux onto a substrate $J$ was calculated for the pure ICP, because the experimental data on $n_{\mathrm{e}}, k T_{\mathrm{e}}$, and $V_{\mathrm{pl}}$ were available; i.e., the electron temperature, the electronic density and the plasma potential were measured respectively $2.6 \mathrm{eV}, 2.8 \times 10^{17} \mathrm{~m}^{-3}$ and $18 \mathrm{~V}$ for the condition of ICP RF Power $200 \mathrm{~W}$ at $30 \mathrm{mT}$ Torr. Thus, we obtained $j$ by substituting the measured values and the physical constants such as $m_{\mathrm{e}}, m_{\mathrm{i}}, E_{\text {rec }}(=15.7 \mathrm{eV}$ for argon ion) into equations (4), (5), and (6). Thus, the theoretical energy flux for $200 \mathrm{~W}$ ICP was calculated against the substrate bias $V_{\text {bias, }}$ which is shown by a solid curve in Fig. 7. The theoretical curve for the 100W ICP was calculated in the same way and it is shown by a short dashed curve in Fig. 7 .

From Fig.7, we notice that 1) the experimental substrate bias dependence of the energy flux is in good agreement with the calculated one for both ICP power 100 and $200 \mathrm{~W}$, 2) experimental energy flux in the pure ICP is almost the same as that in the ICP assisted MP discharge, indicating that the energy flux is dominantly determined by the ICP power. For the detailed analysis, we need to include the contributions of neutral species.

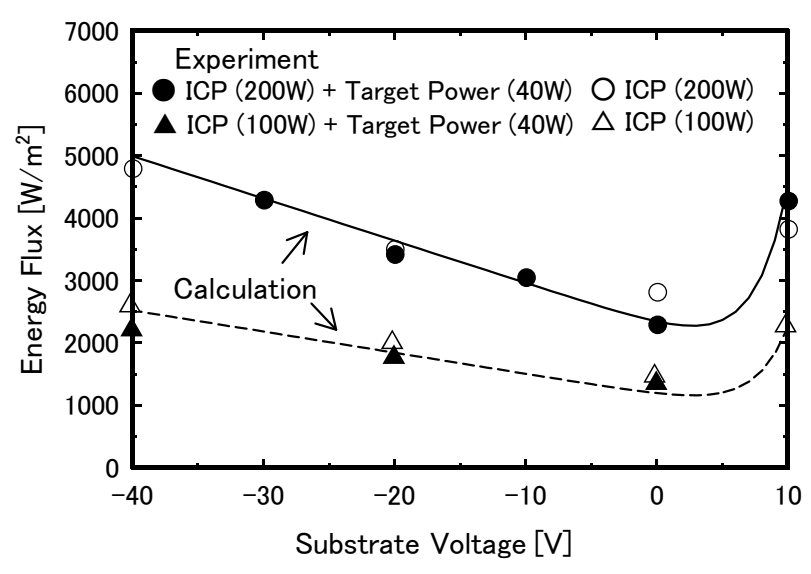

Figure 7. Substrate bias dependence of energy flux: Comparison of measured and calculated energy flux.

\section{CONCLUSION}

Temperature equilibrium type thermal probe operating with a time constant about $100 \mathrm{~s}$ was developed in order to measure the energy flux onto a substrate during ICP assisted sputter-deposition. The energy flux was proportional to the target power in a pure planar magnetron, and it was proportional to the ICP power in a pure ICP. However, the energy flux in the ICP assisted sputtering is not a simple addition of the energy flux in the pure planar magnetron and that in the pure ICP; i.e., the energy flux in the ICP assisted sputtering is predominantly determined by the ICP power. The energy flux measured with the thermal probe was in good agreement with the calculated results, which were based on the model proposed by Kersten et al.

\section{ACKNOWLEDGMENT}

This research was partially supported by a Grant-in-Aid for Scientific Research (C) from the Japan Society for the Promotion of Science (No. 20540485) and by Special Coordination Funds for Promoting Science and Technology sponsored by Japan Science and Technology Agency (JST). 


\section{REFERENCES}

[1] Y. Matsuda, T. Shibasaki, M. Shinohara, "Smomthing of resistivity distributions of sputtered AZO thin flim by the assist of ICP," Proc. of the 25th SPP, Yamaguchi, Japan, January 23-25, 2008, A5-01 (2008) pp.79-80.

[2] H. Kersten, D. Rohde, J. Berndt, H. Deutsch, R. Hippler, "Investigations on the energy influx at plasma processes by means of a simple thermal probe,’Thin Solid Films 377-378 (2000) pp.585-591.

[3] H. Kersten, G. M. W. Kroesen, R. Hippler, "On the energy influx to the substrate during sputter deposition of thin aluminum films," Thin Solid Films 332 (1998) pp.282-289.

[4] H. Kersten, H. Deutsch, H. Steffen, G.M.W. Kroesen, R. Hippler, "The energy balance at substrate surfaces during plasma processing," Vacuum 63 (2001) 385-431.

[5] H. Kersten, D. Rohde, H. Deutsch, R. Hippler W.W. Stoffels, E. Stoffels, G.M.W. Kroesen, J. Berndt, "Investigations on the energy influx at plasma surface processes," acta physica slovaca 50 (2000) pp.439-459.

[6] M. Wolter, M. Stahl, H. Kersten, "Spatially resolved thermal probe measurement for the investigation of the energy influx in an rfplasma," Vaccum (2008) 768-772 .

[7] H. Kersten, E. Stoffels, W. W. Stoffels, M. Otte, C. Csambal, H. Deutsch, R. Hippler, "Energy influx from an rf plasma to a substrate during plasma processing," J. Appl. Phys. 87 (2000) pp.3637-3645.

[8] R. Wendt and K. Ellmer, K. Wiesemann, "Thermal power at a substrate during $\mathrm{ZnO}: \mathrm{Al}$ thin film deposition in a planar magnetron sputtering system,” J. Appl. Phys. 82 (1997) pp.2115 - 2122.

[9] L. Sirghi, G. Popa, Y. Hatanaka, "Heating of polymer substrate by discharge plasma in radiofrequency magnetron sputtering deposition," Thin Solid Films 515 (2006) pp.1334 - 1339.

[10] H. Matsuura, T. Jida, "Determination of Plasma Parameters from Thermal Probe Data,” Contrib. Plasma Phys.46 (2006) pp.406-410. 